\title{
Searching for Critical Slip Surface of Slope Based on Flower Pollination Algorithm
}

\author{
Runying Wang, Keping Yu* \\ College of Water Conservancy and Hydropower Engineering, Hohai University, Nanjing, Jiangsu, 210024, China
}

\begin{abstract}
In order to determine the critical sliding surface of the slope, the most dangerous sliding surface was searched by flower pollination algorithm. First, the method is applied to the traditional vertical slice method for slope stability analysis. The results are very close to those of the Autobank program, indicating that the method can search the critical sliding surface and find the optimal solution. Then, the method is applied to the new horizontal slice method, and the results of the calculation examples are better than those given in the references, indicating that the application of the flower pollination algorithm to the horizontal slice method is reasonable, reliable and superior. The successful application of this method provides a new method for determining the most dangerous sliding surface of slope.
\end{abstract}

\section{Introduction}

Slope stability analysis is of great significance in engineering fields such as pit exploration engineering, highway engineering, water conservancy engineering, etc. It is an important means of engineering safety evaluation. At present, the most widely used method in engineering is the limit equilibrium method. After determining the limit equilibrium method, the key to determine the minimum safety factor is whether the most dangerous sliding surface can be found.

At present, there are many optimization algorithms for searching the most dangerous sliding surface of slope. Xiaofeng Huang et al.[1] used particle swarm optimization theory to determine the position of the most dangerous potential sliding surface. Fei Kang et al.[2] introduced artificial bee colony algorithm into slope stability analysis to determine the potential sliding surface. Chenghua Wang et al.[3] used ant algorithm search technology on the basis of finite element. Xianjun Bai et al.[4] proposed an accelerated genetic algorithm to search the most dangerous sliding surface of slope. Mingrui Zhang et al.[5] introduced the simulated annealing mechanism into the intelligent single particle algorithm to search for the most dangerous sliding surface. These algorithms have achieved good results in the search of the most dangerous sliding surface of slope, but they also have some defects. Genetic algorithm has the problem that the parameters are not easy to be determined. The parameters of simulated annealing algorithm are difficult to be controlled and cannot be guaranteed to converge to the optimal value at one time. Ant algorithm is applied to combinatorial optimization problem, while the most dangerous sliding surface search is a continuous numerical optimization problem. The artificial bee colony algorithm has the defect of slow convergence speed. Particle swarm optimization algorithm is difficult to determine the parameters, convergence speed is too fast to lead to low accuracy. Flower pollination algorithm has the advantages of simple structure, few parameters and strong robustness, and has been widely concerned since it was proposed. In this paper, the flower pollination algorithm is used to search the critical sliding surface of slope to determine the minimum safety factor of slope.

At present, the limit equilibrium method mainly adopts vertical slice method. The common vertical slice methods include Sweden slice method, simplified Bishop method, Janbu method, Morgenstern-Price method. Although the vertical slice method has many advantages, it still has some defects. For example, the horizontal slice method is obviously better than the vertical strip division method in calculating the slope with stratified soil distribution. In this paper, based on the study of the application of flower pollination algorithm to the critical sliding surface search of slope, the critical sliding surface search method is applied to the horizontal slice method, which provides a new optimization algorithm for the application of horizontal slice method, and the most dangerous sliding surface is searched more quickly and accurately.

\section{Solving the most dangerous sliding surface of slope based on pollination algorithm}

\subsection{The most dangerous sliding surface search theory model of slope}

Considering the checking calculation with the existing calculation examples, the Swedish strip division method

\footnotetext{
* Corresponding author: iop234@126.com
} 
in the vertical slice method is used for calculation, and the most dangerous sliding surface search theoretical model of slope is established. The formula for calculating the safety factor of Sweden slice method is as follows:

$$
F_{\mathrm{s}}=\frac{\sum\left(c_{i} l_{i}+W_{i} \cos \alpha_{i} \tan \varphi_{i}\right)}{\sum W_{i} \sin \alpha_{i}}
$$

$\alpha_{i}$ is the bottom slope angle of bar $i . \varphi_{i}$ refers to the angle of internal friction of bar $i . c_{i}$ is the cohesion of bar $i . l_{i}$ is the bottom length of the bar $i$. $W_{i}$ is the weight of the bar $i . F_{\mathrm{s}}$ is the safety factor of the sliding surface.

Set the circular center coordinate $(x, y)$ and the radius $r$ of the circular sliding surface as design variables. The most dangerous sliding surface search problem can be translated into the following optimization problem:

$$
\min F_{\mathrm{s}}=f(x, y, r)
$$

\subsection{Flower pollination algorithm}

The flower pollination algorithm originates from the phenomenon of flower pollination in nature. The act of pollination allows plants to reproduce. In nature, there are two kinds of pollination, self-pollination and crosspollination. Among them, cross-pollination plays a dominant role in flower pollination. Cross-pollination occurs in two flowers that are far apart, while selfpollination occurs in monoecious flower plants. The global search in the algorithm corresponds to crosspollination in nature, while the local search corresponds to self-pollination in nature. Switching probability is used to balance the relationship between global search and local search, so that the algorithm search performance is better. The flower pollination algorithm mainly contains the following several main steps[6]:

(1) Initialization parameters: pollen population size $N$, search spatial dimension $D$, switching probability $P$, maximum iteration times of the population IterMax.

(2) The pollen population was randomly generated, the optimal location $g_{*}$ of the initial random solution was found, and the fitness value $f\left(g_{*}\right)$ was calculated.

(3) If rand $<\mathrm{P}$ (rand is a random parameter that is uniformly distributed $[0,1]$ ), the algorithm enters the main loop to conduct the global search process,

$$
\begin{gathered}
x_{i}^{t+1}=x_{i}^{t}+L\left(x_{i}^{t}-g_{*}\right) \\
L \sim \frac{\lambda \Gamma(\lambda) \sin (\pi \lambda / 2)}{\pi} \frac{1}{s^{1+\lambda}}, \quad\left(s \gg s_{0} \gg 0\right)
\end{gathered}
$$

$x_{i}^{t+1}$ is the position of pollen at time $t+1 . g_{*}$ is the current optimal solution position of the population. $L$ is the control parameter. $\Gamma(\lambda)$ as the standard gamma function, take $1.5 \lambda$.

Update current pollen location.

If rand $>\mathrm{P}$, the local search process is performed,

$$
x_{i}^{t+1}=x_{i}^{t}+\varepsilon\left(x_{j}^{t}-x_{k}^{t}\right)
$$

$x_{j}^{t}$ and $x_{k}^{t}$ are the locations of two pollen different from $i$ in the population. $\varepsilon$ is a proportional coefficient that follows a uniform distribution $[0,1]$.

Also updates the current pollen location.

(4) Analyze whether the individual needs to be updated. If $f\left(x_{i}^{t+1}\right)<f\left(x_{i}^{t}\right)$, update solution will simultaneously pollinate $x_{i}^{t}$ to the new position $x_{i}^{t+1}$.

(5) Compare it to the optimal pollen. If $f\left(x_{\text {new }}\right)<f\left(g_{*}\right)$, $x_{\text {new }}$ will replace the optimal solution $g_{*}$.

(6) Judge whether the current pollen is the last pollen in the population. If so, enter the next step. If not, return step (4).

(7) Judge whether the algorithm has reached the set maximum number of iterations. If it has, it will enter the next step. If not, it will return step (3) and continue to enter the next generation of search.

(8) When the algorithm is finished, it outputs the calculated optimal position $g_{*}$ and the optimal solution $f\left(g_{*}\right)$.

For the optimization problem of solving the slope's most dangerous sliding surface, $(x, y, r)$ is the position of pollen, and $F_{\mathrm{S}}$ is the fitness value. Finally, the optimal center coordinates and radius of the circle are obtained, and the optimal solution is the minimum $F$ s.

\section{Horizontal slice method and its most dangerous sliding surface determination}

Similar to the vertical slice method, the horizontal slice method also divides the sliding soil into bars. But unlike the vertical slice method, the bars in horizontal slice method are horizontal. Therefore, compared with the vertical slice method, the horizontal slice method is more simplified and the calculation results are more reasonable when calculating the slope with stratified soil distribution, the slope under seismic conditions and the slope with groundwater. In this paper, based on the Swedish theory of bar division, the method of horizontal slice method is calculated by using the form of integral instead of the usual method of bar division. Figure 1 shows the force analysis of the horizontal bar section. In the figure, $\mathrm{O}$ is the center of the sliding surface, $\mathrm{d} M$ is the sliding moment infinitesimal, $\mathrm{d} w$ is the gravity infinitesimal, $\mathrm{d} N$ is the normal reaction force of the sliding surface, $\mathrm{d} T$ is the anti-sliding force, and $\theta$ is the angle between the arc length and the horizontal line.

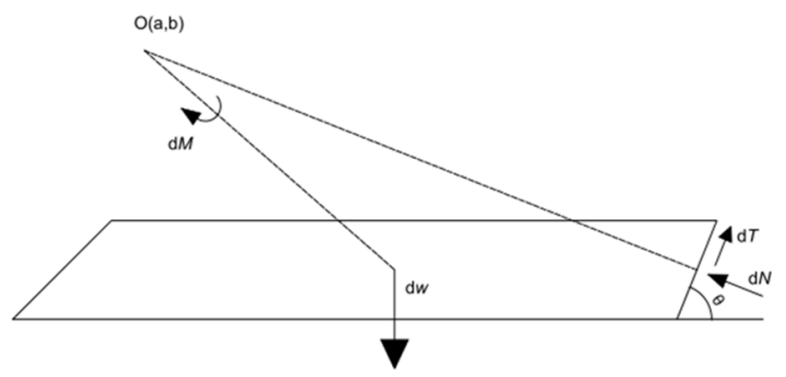

Fig. 1. Force analysis of horizontal slice model.

Similarly, the circular center coordinate $(a, b)$ and the radius $r$ of the circular sliding surface are set as design 
variables. The most dangerous sliding surface search problem can be translated into the following optimization problem:

$$
\min F_{\mathrm{s}}=f(a, b, r)
$$

When $r \leq \sqrt{a^{2}+b^{2}}$, the slope failure mode is shown in figure 2 (a). When $r>\sqrt{a^{2}+b^{2}}$, the failure mode of the slope is shown in figure 2 (b). In the figure, $\mathrm{O}$ is the center of the sliding surface, $r$ is the radius of the arc, $h$ is the slope height, and $\beta$ is the slope angle.

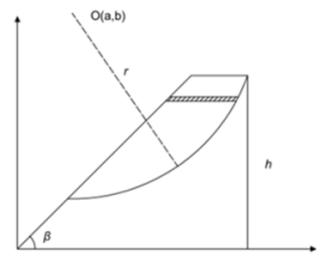

(a)

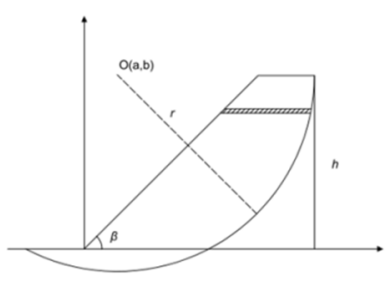

(b)
Fig.2. Slope failure model.

According to the limit equilibrium theory, the calculation formula of safety coefficient is,

$$
F_{\mathrm{s}}=\frac{M_{f}}{M}=\frac{\tau_{f} \times L \times r}{\tau \times L \times r}
$$

$\tau_{f}$ is the shear strength of sliding surface. $\tau$ is the shear stress on the sliding surface. $L$ is the sliding arc length. $r$ is the radius of sliding arc.

The overall stability coefficient of the sliding surface using the horizontal slice method is[7] :

When $r \leq \sqrt{a^{2}+b^{2}}$

$$
\begin{aligned}
M_{f}= & \gamma \tan \varphi\left\{\frac{2 h^{3}-3 b h^{2}}{6 \tan \beta}+\frac{\left[r^{2}-(h-b)^{2}\right]^{1.5}}{3}+\frac{a\left[b^{2}-(h-b)^{2}\right]}{2}\right. \\
& \left.-\frac{\left(r^{2}-b^{2}\right)^{1.5}}{3}\right\}+c r^{2}\left(\arcsin \frac{b}{r}+\arcsin \frac{h-b}{r}\right) \\
& M=\gamma\left\{\frac{3 a \tan \beta h^{2}-h^{3}}{6 \tan ^{2} \beta}+\frac{h\left(r^{2}-a^{2}\right)}{2}-\frac{(h-b)^{3}+b^{3}}{6}\right\}
\end{aligned}
$$

When $r>\sqrt{a^{2}+b^{2}}$

$$
\begin{aligned}
M_{f}= & \gamma \tan \varphi\left\{\frac{2 h^{3}-3 b h^{2}}{6 \tan \beta}+\frac{\left[r^{2}-(h-b)^{2}\right]^{1.5}}{3}+\frac{a\left[b^{2}-(h-b)^{2}\right]}{2}\right. \\
& \left.+\frac{\left(r^{2}-b^{2}\right)^{1.5}}{3}\right\}+c r^{2}\left(\arcsin \frac{h-b}{r}-\arcsin \frac{b}{r}+\pi\right) \\
& M=\gamma\left\{\frac{3 a \tan \beta h^{2}-h^{3}}{6 \tan ^{2} \beta}+\frac{h\left(r^{2}-a^{2}\right)}{2}-\frac{(h-b)^{3}+b^{3}}{6}\right\}
\end{aligned}
$$

\section{The example analysis}

\subsection{Example analysis of vertical slice method}

The parameters in reference[8] were used for calculation, and Autobank program of Hohai University was used for checking calculation. Unit weight $\gamma=18.8 \mathrm{kN} / \mathrm{m}^{3}$, slope height $h=46 \mathrm{~m}$, slope ratio 2.25 , sticky cohesion $c=$ $48.0 \mathrm{kPa}$, internal friction angle $=12.5^{\circ}$. The calculation results are shown in table 1.

Table 1. Comparison of calculation results.

\begin{tabular}{|c|c|c|c|c|}
\hline Calculation method & $\boldsymbol{x} / \mathbf{m}$ & $\boldsymbol{y} / \mathbf{m}$ & $\boldsymbol{r} / \mathbf{m}$ & $\boldsymbol{F}_{\mathbf{s}}$ \\
\hline Reference & 30.94 & 107.01 & 111.44 & 1.07 \\
\hline $\begin{array}{c}\text { Flower pollination } \\
\text { algorithm }\end{array}$ & 33.35 & 85.78 & 93.09 & 1.06 \\
\hline Autobank & 33.58 & 87.02 & 94.50 & 1.06 \\
\hline
\end{tabular}

According to the calculation results in table 1 , the safety coefficient obtained by the three calculation methods is very close, and the position of the most dangerous slide surface calculated by the method in this paper is more similar to that calculated by Autobank, which indicates that the most dangerous slide surface search based on the flower pollination algorithm proposed in this paper is reliable.

\subsection{Example analysis of horizontal slice model}

The parameters in reference [7] were used for calculation, and the Autobank program of Hohai University was also used for checking and comparison with vertical slice method. Unit weight $\gamma=18 \mathrm{kN} / \mathrm{m}^{3}$, slope height $h=6 \mathrm{~m}$, slope angle $\beta=60^{\circ}$, sticky cohesion $c=20 \mathrm{kPa}$, internal friction angle $=22^{\circ}$. The calculation results are shown in table 2 .

Table 2. Comparison of calculation results.

\begin{tabular}{|c|c|c|c|c|}
\hline Calculation method & $\boldsymbol{x} / \mathbf{m}$ & $\boldsymbol{y} / \mathbf{m}$ & $\boldsymbol{r} / \mathbf{m}$ & $\boldsymbol{F}_{\mathbf{s}}$ \\
\hline Reference & -3 & 10 & 10.44 & 1.48 \\
\hline $\begin{array}{c}\text { Flower pollination } \\
\text { algorithm }\end{array}$ & 0.04 & 6.07 & 6.07 & 1.39 \\
\hline Autobank & -0.11 & 7.86 & 8.26 & 1.82 \\
\hline
\end{tabular}

It can be seen from the calculation results in table 2 that more dangerous sliding surfaces can be searched by using the flower pollination algorithm, and the calculation results are more reliable. Moreover, the calculation results of horizontal slice model are smaller than that of vertical slice model. The results show that the proposed horizontal slice model based on flower pollination algorithm is reliable.

\section{Conclusion}

In this paper, flower pollination algorithm is used to search for the most dangerous sliding surface, and this method is applied to horizontal slice method. Combined 
with the calculation results, the following conclusions are drawn:

The flower pollination algorithm is very effective in solving continuous variable optimization problems. It has the characteristics of simple structure, few parameters and strong robustness.

In this paper, the flower pollination algorithm is successfully applied to the most dangerous sliding surface search using the traditional vertical slice model. The calculated results are similar to those of the Autobank program and better than those given in the references. It shows that the flower pollination algorithm is accurate and reliable for the most dangerous sliding surface search of slope.

The flower pollination algorithm is successfully applied to a novel horizontal slice model, which provides a reference for determining the most dangerous sliding surface of horizontal slice model. Compared with the results given in the references, the flower pollination algorithm can search more dangerous sliding surfaces and find the minimum safety coefficient, which proves the superiority and reliability of the method, and provides a new method for solving the problem of determining the most dangerous sliding surfaces by horizontal slice model.

\section{References}

1. Huang, X.F., Shi, C., Zhu, Z.D., Wang S.N., Zhang, R.C. (2014) Determination Method of Critical Slip Surface Based on PSO Algorithm. Journal of Disaster Prevention and Mitigation Engineering, 34(06):751-757.

2. Kang, F., Li, J.J., Ma, Z.Y. (2011) Searching for Critical Slip Surface of Slops Based on Artificial Bee Colony Algorithm. Journal of Disaster Prevention and Mitigation Engineering, 31(02):166172.

3. Wang, C.H., Xia, X.Y., Li, G.X. (2003) Ant Algorithm in Search of The Critical Slip Surface in Soil Slopes Based on Stress Fields. Chinese Journal of Rock Mechanics and Engineering, (05):813-819.

4. Bai, X.J., Li, X.X. (2011) A new method to search the most dangerous sliding surface of slope and its corresponding minimum safety factor. Inner Mongolia Water Resources, (06):10-11.

5. Zhang, M.R., Chen, Z.Z., Liu, Y.H., Cao, D.F., Wu, Q. (2011) Application of Intelligent Single Particle Optimizer with Simulated Annealing Mechanism to Searching for Critical Slip Surface of Complex Slope. Journal of Engineering Geology, 19(02):181186.

6. Chen, Q., Xie, C.N., Xing, J.G., Zhao, H. (2020) An Improved Optimization Algorithm for Flower Pollination. Mechanical \& Electrical Engineering Technology, 49(07):211-213+224.

7. Lu, Y.L., Bo, J.S., Chen, X.R. (2017) Error Analysis of a Horizontal Slice Model Using the Swedish Arc Method. China Earthquake Engineering Journal, 39(03):496-501.
8. Hu, H. (2017) The analytical calculation of slope stability analysis based on Matlab (Swedish bar division method). Urban Roads Bridges \& Flood Control, 000(009):198-199. 